\title{
Reach out and touch someone: potential impact of DIET (direct interspecies energy transfer) on anaerobic biogeochemistry, bioremediation, and bioenergy
}

\author{
Derek R. Lovley
}

Published online: 5 May 2011

(C) Springer Science+Business Media B.V. 2011

Tell me what you eat, and I'll tell you what you are.

Anthelme Brillat-Savarin Most prokaryotes are unicellular and are typically studied as a collection of independently functioning cells. Even when two species of microorganisms need to cooperate in order to survive, the two species are usually regarded as separate entities. This is also true when microbes grow in biofilms or multi-cellular aggregates; the individual cells are modeled as bricks in the overall structure and sharing of metabolites, or conveyance of information via signaling molecules, is assumed to rely on the release of these substances into the extracellular matrix. However, exceptions in which cellular contact is required for signaling are being increasing recognized (Marx 2009).

In a similar manner, recent studies have suggested that the exchange of energy between microbial species may be much more sophisticated and effective than previously considered. In particular, direct interspecies energy transfer (DIET) may be an important feature of microbial communities that play key roles in the global cycling of carbon and

D. R. Lovley $(\varangle)$

Department of Microbiology, University of Massachusetts

Amherst, Amherst, MA, USA

e-mail: dlovely@microbio.umass.edu nutrients, the remediation of environmental contaminants and various bioenergy strategies.

For example, one of the best-known examples of interspecies cooperation is interspecies hydrogen transfer, which is a strategy for microorganisms to exchange electrons (McInerney et al. 2009; Stams and Plugge 2009). A microorganism that needs to release electrons, derived from the oxidation of an organic substrate, reduces protons with those electrons, producing hydrogen gas. A microorganism that can gain energy from the oxidation of the hydrogen then consumes it. Since first proposed over 40 years ago (Bryant et al. 1967), interspecies hydrogen transfer has been considered to be central to the production of methane gas in anaerobic soils, sediments, and animal intestinal systems (McInerney et al. 2009; Stams and Plugge 2009). The positive benefit is effective anaerobic metabolism of organic matter in these environments. A potential negative environmental consequence is that methane is a potent greenhouse gas. Conversion of wastes to methane in digesters, also presumed to rely on interspecies hydrogen transfer, is an important bioenergy strategy.

It can clearly be shown in the laboratory that microorganisms that produce hydrogen from the anaerobic degradation of various organic substrates can grow in co-culture with hydrogen-consuming methanogens and, in fact, the hydrogen-producers generally require the methanogens to remove hydrogen in order to make continued hydrogen production 
thermodynamically feasible (McInerney et al. 2009; Stams and Plugge 2009). Formate can substitute for hydrogen as an electron carrier in some of these cultures (McInerney et al. 2009; Stams and Plugge 2009). However, for a variety of technical reasons, proving that interspecies hydrogen/formate transfer is important in natural methanogenic environments has been problematic (Ozturk et al. 1989).

Now, it is apparent that another possibility must also be considered. In a study initially designed to evaluate how microorganisms might evolve for effective interspecies hydrogen transfer, it was found that some microorganisms may exchange electrons through direct electrical connections, rather than producing hydrogen or formate as electron carriers (Summers et al. 2010). Two Geobacter species, placed under conditions in which they were required to share electrons in order to metabolize ethanol, evolved to form electrically conductive aggregates of the two species, as they adapted for more rapid ethanol metabolism. Details on the nature of the electrical connection are still to be elucidated, but electrically conductive pili, known as microbial nanowires (Reguera et al. 2005), as well as a $c$-type cytochrome that may facilitate electron transfer to or from the pili (Leang et al. 2010), appear to be involved (Summers et al. 2010). Cell-to-cell electron transfer via microbial nanowires had previously been proposed, based on apparent connections between cells via filaments (Reguera et al. 2005; Gorby et al. 2006; Reguera et al. 2006), but only with a genetically tractable co-culture was it possible to experimentally evaluate this possibility.

Cell aggregation is a common phenomenon in defined methane-producing cultures (Johnson et al. 2006; Schopf et al. 2008; Stams and Plugge 2009), as well as in more complex systems such as waste digesters (Schmidt and Ahring 1996). Aggregation can be considered as a strategy to promote more effective exchange of hydrogen or formate, but it also makes direct electron transfer feasible. In fact, methanogenic aggregates from wastewater digesters were found to be electrically conductive, consistent with direct conduction of electrons between cells (Morita et al. 2011). Conductivity was associated with an abundance of Geobacter species, that were presumably functioning as syntrophic fatty acid oxidizers in the natural aggregates. It seems likely that microorganisms other than Geobacter species may be able to make electrical contacts in anaerobic environments (Gorby et al. 2006; Summers et al. 2010), but this has yet to shown experimentally.

Aggregates anaerobically oxidizing methane with the reduction of sulfate are another likely candidate for direct interspecies electrical connections (Alperin and Hoehler 2010). A series of experimental and modeling studies have suggested that hydrogen, formate, or a diversity of other potential soluble electron shuttles, are unlikely electron carriers between the archaea responsible for the initial attack on methane and their companion sulfate reducers (Orcutt and Meile 2008; Alperin and Hoehler 2010; Meulepas et al. 2010). At least one clade of these methane-oxidizing aggregates expresses genes encoding multi-heme $c$-type cytochromes that are predicted to be excreted from cells (Myerdierks et al. 2010), and thus analogous to cytochrome required for direct electron transfer between Geobacter species (Summers et al. 2010). Measurements on the conductance of anaerobic methane-oxidizing aggregates should help resolve whether direct electron transfer within this microbial assemblage is feasible.

Electrons are just one example of energy currency that different microbial species might want to exchange. Metabolites are also important. When one species of a cooperative consortium releases a metabolite into the extracellular matrix there are no guarantees that it will reach its cooperative partner species. Extracellular enzymes may degrade the metabolite, an unwanted competitor may take up the metabolite, or it may diffuse far away from the microbial assemblage before it can be consumed by the target species. Unlike electrons, metabolites cannot be transmitted through microbial nanowires. However, they may be directly transferred from one species to another via recently described structures known as intercellular nanotubes (Dubey and BenYehuda 2011). In studies with Bacillus subtilis, the nanotubes, which appeared to make connections between the cytoplasms of cells, formed only when cells were grown on solid surfaces. When a strain of B. subtilis expressing an introduced gene for the production of a cytoplasmic fluorescent protein was grown next to a strain of $B$. subtilis that did not produce the protein, the protein migrated from the producer cells to the non-producer cells. Highresolution images of fluorescent molecules, localized with gold-labeled antibodies, showed fluorescent 
protein moving through the nanotubes from one cell to another. Enzymes and DNA could also be transferred from cell to cell, presumably via the same conduits. Connections between multiple cells were noted. Intercellular nanotubes, facilitating cytoplasmic exchange between B. subtilis and Staphylococcus aureus or Escherichia coli, were also documented (Dubey and Ben-Yehuda 2011). An appropriate term for such structures may be interspecies nanotubes. The degree of exchange of cytoplasmic constituents between cells via interspecies nanotubes has yet to be quantified, but these initial studies clearly raise the possibility for two microbial species, with distinctly different metabolic capabilities, to cooperatively act as a single super organism by efficiently sharing metabolites in a highly coordinated manner.

Structures analogous to interspecies nanotubes are found in the symbiotic association known as "Chlorochromatium aggregatum" (Wanner et al. 2008), an assemblage of a central heterotrophic bacterium surrounded by multiple green sulfur bacteria. The green sulfur bacteria are thought to provide the central bacterium with organic carbon, whereas the central bacterium provides motility to the consortium, allowing it to orient at the depths at which light wavelengths are optimal for sulfur-based photosynthesis. The constituents of the consortium have a number of novel structures associated with their symbiosis. The most intriguing of these may be the connections between the cells, termed 'periplasmic tubules', which appear to connect the periplasms of the two cooperating species. It has not yet been demonstrated what types of materials may be exchanged via the tubules, but it is tempting to speculate that they may function similar to interspecies nanotubes, permitting a diversity of molecules to move between the two species.

Given the intriguing possibilities of DIET, in the form of interspecies electron transfer via microbial nanowires or interspecies metabolite exchange via nanotubes, the important question is just how important DIET is in the environment. Answering this will require carefully designed measurements in natural communities within the relevant environments, as well as controlled laboratory studies with defined consortia. Environmental studies require rigor. For example, the intriguing speculation that electron conduction through marine sediments might proceed via microbial nanowires has yet to be substantiated with direct evidence and many alternative explanations are possible (Nielsen et al. 2010).

One reason that DIET has not been widely observed previously might be that the methods commonly employed for enrichment and isolation of microorganisms that participate in syntrophic associations typically select for microorganisms that interact via release of molecules into the extracellular environment. There can be a selection against clustering when fast, inefficient metabolism is favored (Pfeiffer and Bonohoeffer 2003). The fastest-growing microbes generally win in most enrichment and isolation protocols.

Microbiologists generally like homogenous cultures and the natural tendency to disperse cultures and keep them suspended by shaking them is unlikely to favor the growth of multi-species aggregates that directly exchange materials. Growth on solid surfaces, while avoiding the traditional route of establishing colonies from single cells, may aid in recovering microorganisms that specialize in DIET. Strategies, such as Magneto-FISH, that make it feasible to separate, identify and obtain the genome sequences of microorganisms that are co-aggregated in natural environments (Orphan 2009) are likely to aid in designing the appropriate selective medium for their growth. It would not be surprising if it is found that organisms, that primarily make a living from DIET, account for some of the abundant but as-yetuncultured microbes that inhabit anaerobic environments.

As more is learned from model systems about specific genes and proteins whose expression is associated with the establishment of cell-to-cell connections for DIET, it will be feasible to better document this phenomenon with sensitive environmental transcriptomic and proteomics approaches. High resolution imaging techniques might be used to identify interspecies nanotube connections and document the metabolites moving between species in environmental samples.

The prevalence of DIET is likely to depend greatly on the extent to which establishing direct connections between cells is the most optimal response to given environmental conditions. For example, direct electron exchange might be expected to be favored when the overall energy yield from a particular reaction is low. The production of hydrogen from reduced electron carriers in the electron-donating organism 
and the oxidation of hydrogen in the electronaccepting partner require multiple enzymatic steps with energy losses associated with each reaction. Depending upon the number and nature of electron transfer steps, direct cell-to-cell electron transfer might better conserve energy, as electrons are transferred from reduced co-factors in the electrondonating cells to electron acceptors in the electronaccepting cells. It was clear in the co-culture study with Geobacter species (Summers et al. 2010) that the adaptation for direct cell-to-cell electron transfer resulted in much faster cell growth, consistent with the concept of higher energy conservation via DIET. However, further investigation of growth yields with other defined co-cultures is required to determine whether this is a general phenomenon. It will also be of interest to document growth benefits of metabolite exchange via interspecies nanotubes.

There are potential disadvantages to establishing direct contacts with another species under some conditions. Substantial aggregation hinders motility of the aggregated cells and their ability to move to new energy sources (Pfeiffer and Bonohoeffer 2003). This may not be a significant constraint in environments such as wastewater digesters or methane seeps at the bottom of the ocean where there is a steady, abundant supply of electron donors. However, in an environment such as a sandy aquifer, where the particulate sources of buried organic matter that fuel microbial metabolism are heterogeneously dispersed, cells might find it more advantageous to maintain motility to search for electron donors and hope that the appropriate syntrophic partner will also show up, rather than to physically attach themselves to their syntrophic partner. In general, when electron acceptors such as oxygen, nitrate, sulfate, or Fe(III) are available, there is less need to develop DIET strategies because the high energy yields from using these electron acceptors permit microorganisms to more completely metabolize organic electron donors.

Genome-scale metabolic modeling can provide important insights into the metabolism of previously understudied microorganisms (Mahadevan et al. 2011) and has already been employed to explore the benefits of hydrogen versus formate as an electron carrier in a methanogenic co-culture (Stolyar et al. 2007). Further development of this approach could be a powerful tool to help develop a better theoretical understanding of the cost/benefit of DIET.
In summary, when the goal is modeling microbial responses to environmental change or manipulating microbial communities for various bioremediation or bioenergy applications, understanding how microbes cooperate to metabolize organic substrates may be just as important as knowing what they consume; DIET could be as significant as diet. It is expected that DIET will be a field of intense investigation in the fields of biogeochemistry, bioremediation, and bioenergy for some time to come.

Acknowledgments Research in my laboratory on DIET has been supported by the Office of Science (Office of Biological and Environmental Research), U.S. Department of Energy Cooperative Agreement DE-FC02-02ER63446 and Award DEFC-0004485.

\section{References}

Alperin M, Hoehler T (2010) The ongoing mystery of sea-floor methane. Science 329:288-289

Bryant MP, Wolin EA, Wolin MJ, Wolfe RS (1967) Methanobacillus omelianskii, a symbiotic association of two species of bacteria. Arch Microbiol 59:20-31

Dubey GP, Ben-Yehuda S (2011) Intercellular nanotubes mediate bacterial communication. Cell 144:590-600

Gorby YA, Yanina S, McLean JS, Rosso KM, Moyles D, Dohnalkova A et al (2006) Electrically conductive bacterial nanowires produced by Shewanella oneidensis strain MR-1 and other microorganisms. Proc Natl Acad Sci USA 103:11358-11363

Johnson MR, Conners SB, Montero CI, Chou CJ, Shockley KR, Kelly RM (2006) The Thermotoga maritima phenotype is impacted by syntrophic interactions with Methanococcus jannaschii in hyperthermophilic coculture. Appl Environ Microbiol 72:811-818

Leang C, Qian X, Mester T, Lovley DR (2010) Alignment of the $c$-type cytochrome OmcS along pili of Geobacter sulfurreducens. Appl Environ Microbiol 76:4080-4084

Mahadevan R, Palsson BO, Lovley DR (2011) In Situ to in silico and back: elucidating Geobacter physiology and ecology with genome-scale modeling. Nat Rev Microbiol 9:39-50

Marx CJ (2009) Getting in touch with your friends. Science 324:1150-1151

McInerney MJ, Sieber JR, Gunsalus RP (2009) Syntrophy in anaerobic global carbon cycles. Curr Opin Biotechnol 20:623-632

Meulepas RJW, Stams AJM, Lens PNL (2010) Biotechnological aspects of sulfate reduction with methane as an electron donor. Rev Environ Sci Biotechnol 9:59-78

Morita M, Malvankar NS, Franks AE, Summers ZM, Giloteaux L, Rotaru AE et al. (2011) Evidence for direct cell-to-cell electron transfer in anaerobic methanogenic wastewater aggregates (manuscript submitted) 
Myerdierks A, Kube M, Kostadinov I, Teeling H, Glockner FO, Reinhardt R, Amann R (2010) Metagenome and mRNA expression analyses of anaerobic methanotrophic archaea of the ANME-1 group. Environ Microbiol 12: 422-439

Nielsen LP, Risgaard-Petersen N, Fossing H, Christensen PB, Sayama M (2010) Electric currents couple spatialy separated biogeochemical processes in marine sediment. Nature 463:1071-1074

Orcutt B, Meile C (2008) Constraints on mechanisms and rates of anaerobic oxidation of methane by microbial consortia: process-based modeling of ANME-2 archaea and sulfate reducing bacteria interactions. Biogeosciences 5: 1587-1599

Orphan VJ (2009) Methods for unveiling cryptic microbial partnerships in nature. Curr Opin Microbiol 12:231-237

Ozturk SS, Palsson BO, Thiele JH (1989) Control of interspecies electron transfer flow during anaerobic digestion: dynamic diffusion reaction models for hydrogen gas transfer in microbial flocs. Biotechnol Bioeng 33:745-757

Pfeiffer T, Bonohoeffer S (2003) An evolutionary scenario for the transition to undifferentiated multicellularity. Proc Natl Acad Sci USA 100:1095-1098

Reguera G, McCarthy KD, Mehta T, Nicoll JS, Tuominen MT, Lovley DR (2005) Extracellular electron transfer via microbial nanowires. Nature 435:1098-1101
Reguera G, Nevin KP, Nicoll JS, Covalla SF, Woodard TL, Lovley DR (2006) Biofilm and nanowire production leads to increased current in Geobacter sulfurreducens fuel cells. Appl Environ Microbiol 72:7345-7348

Schmidt JE, Ahring BK (1996) Granular sludge formation in upflow anaerobic sludge blanket (UASB) reactors. Biotechnol Bioeng 49:229-246

Schopf S, Wanner G, Rachel R, Wirtth (2008) An archaeal bi-species biofilm formed by Pyrococcus furiosus and Methanopyrus kandleri. Arch Microbiol 190:371-377

Stams AJ, Plugge CM (2009) Electron transfer in syntrophic communities of anaerobic bacteria and archaea. Nat Rev Microbiol 7:568-577

Stolyar S, Van Dien S, Hillesland KL, Pinel N, Lie TJ, Leigh JA, Stahl DA (2007) Metabolic modeling of a mutalistic microbial community. Mol Syst Biol 3:92

Summers ZM, Fogarty H, Leang C, Franks AE, Malvankar NS, Lovley DR (2010) Direct exchange of electrons within aggregates of an evolved syntrophic co-culture of anaerobic bacteria. Science 330:1413-1415

Wanner G, Vogl K, Overmann J (2008) Ultrastructural characterization of the prokaryotic symbiosis in "Chlorochromatium aggregatum". J Bacteriol 190:3721-3730 